\title{
Virtualidad: Una Oportunidad para acercar la Educación Continúa a nuestros egresados.
}

Virtuality: An opportunity for approach our graduates to continuing education.

Filiación:

1.Terapeuta Física; Especialista en Rehabilitación Funcional de la Persona Adulta Mayor. Directora, investigadora y Docente Escuela de Terapia Física Universidad Santa Paula, San José, Costa Rica

\section{Correspondencia: $\square$}

Erika Cyrus Barker

ericyrus19@gmail.com

\section{Filiación:}

2.Terapeuta Física, Especializada en Terapia Manual Ortopédica, Docente de Kinesiología Universidad Santa Paula, San José, Costa Rica

Correspondencia: $\square$

Carolyn Ramer Mesén

cjramer73@gmail.com

Forma de citar: Cyrus Baker E, Ramer Mesén C. Virtualidad: una oportunidad para acercar la Educación Continua a nuestros egresados. Rev Ter. 2017;11(2):4-6.

Financiamiento:

Autofinanciado

Conflictos de Interés:

El autor declara no tener conflictos de interés en la presente publicación
Erika Cyrus Barker '; Carolyn Ramer Mesén $2 \square$

\section{Resumen.}

Ante los retos actuales que las profesionales de la salud se encuentran expuestas, la educación continua plantea una solución viable, sin embargo, es necesario hacerla accesible en tiempo y espacio, considerando las responsabilidades, necesidades y tiempos delos posibles estudiantes, es por esto que se plantea la virtualidad como el medio ideal para permitir lograr afrontar los retos y brindar oportunidades de actualización y educación continua a nuestros estudiantes y egresados

Palabras claves: educación continua, virtualidad, acceso, actualización, recursos

\section{Abstract.}

Given the current challenges that health professionals are exposed, continuing education poses a viable solution, however, is necessary to make it accessible in time and space, considering the responsibilities, needs and times prospective students, is why virtuality arises as to allow achieve the ideal address the challenges and opportunities of renovation providing medium and continuing education to our students and graduates

Key words: continuing education, virtual, access, update, resources

La educación continua está presente en un gran número de países y muestra cada día un fuerte avance, pues lejos de detenerse en los escenarios educativos locales, su adecuada gestión ha permitido su extensión a espacios internacionales como los congresos bianuales que realiza nuestra escuela. No obstante las buenas intenciones de algunas instituciones educativas por cubrir las necesidades de la sociedad en su área de competencia, han proliferado actos académicos que ofrecen oportunidades de actualización aprovechando el mercado que se presenta día a día, sin consideración a los principios de planeación 
y evaluación respectivos y al perfil de la población a los que van dirigidos dichos procesos ${ }^{1,2}$. Es por esto que es nuestra responsabilidad como institución que se dedica al desarrollo humano poder de manera responsable ofrecer opciones de educación continua a nuestros estudiantes y egresados así como a la sociedad en general

La Escuela de Terapia Física consiente es consciente de la responsabilidad de mantener a sus egresados con posibilidades de acceso a la capacitación y educación continua, para tal efecto se trabaja de manera constante en el desarrollo e implementación de un programa de educación continua, atractivo, que supla las necesidades de actualización y desarrollo académico de la población meta.

Sin embargo tenemos un problema por resolver: y es el acceso a este programa por personas que viven lejos, o por sus responsabilidades cuentan con menor cantidad de tiempo.

A pesar de encontrarnos en la era de la globalización, en nuestra universidad la totalidad de los cursos son dirigidos a los estudiantes que pueden dedicar su tiempo presencial para los mismos, sin tomar en cuenta a la población de egresados que vive fuera de la gran área metropolitana (GAM), los cuales desean ser parte de las actividades de educación continua y actualización sin embargo por distancias, tiempo y dinero se les hace imposible participar. Es ahí donde la educación virtual rompe la barrera y permite el acceso a todos los interesados, flexibilizando tiempos ya que el estudiante puede estudiar a la hora que le convenga, el estudiante se vuelve un ente activo, protagonista de su propio aprendizaje y puede tomar los cursos de actualización cuando los necesite sin desatender sus obligaciones laborales o personales ${ }^{4-5}$. Otra ventaja es la posibilidad de contar con docentes expertos que por las mismas dificultades temporales y espaciales no se podrían contratar. Los egresados son el termómetro en la sociedad de la calidad educativa que brindamos en nuestras aulas, para nadie es un secreto que en el campo de la salud los avances y descubrimientos de nuevas metodologías y técnicas llevan un ritmo vertiginoso, no podemos dejar al egresado desprovisto de tales herramientas, por lo que consideramos que es deber del alma mater brindarles opciones de capacitación y educación continua, sino estarán en clara desventaja con los nuevos egresados y no podrán satisfacer las demandas y necesidades que la sociedad les impone.

La Educación Virtual es una propuesta de educación no presencial a través de Internet accesible desde cualquier lugar y a cualquier hora, este sistema de educación tiene la ventaja en que los alumnos y los profesores no están en el mismo lugar

Esta modalidad se orienta a la utilización más flexible de los recursos académicos, de tiempo y espacio y ofrece a los alumnos la posibilidad de administrar libremente sus tiempos de estudio y trabajo.

Las estrategias empleadas y la riqueza que ofrece esta modalidad en medios de comunicación permiten transmitir información de carácter cognoscitivo y mensajes formativos, sin la necesidad de establecer una relación permanente de carácter presencial y circunscrito a un espacio específico ${ }^{6-7}$.

Además ofrece la posibilidad de fomentar en el estudiante la capacidad de autoformación dado que desaparece la instrucción tradicional, dando paso a una educación centrada en el estudiante y no en el profesor como ha sido lo habitual.

Dado que las formas y posibilidades educativas han evolucionado a pasos agigantados y tomando en cuenta que nuestra Universidad es pionera en todas las carreras que imparte, no podemos quedarnos atrás en el campo de la virtualidad. Es imperativo que demos el salto, capacitemos a los docentes e iniciemos la aventura de abrirnos al mundo de posibilidades que la virtualidad ofrece3, la propuesta de este proyecto es el primer ejercicio formal en 
este campo y de este dependerá la apertura que la administración (al final sin el apoyo económico no podremos avanzar mucho) y la academia tengan ante estas nuevas propuestas educativas ${ }^{7}$.

Si bien es cierto la Escuela de Terapia Física cuenta con un programa de actualización y educación continua de avanzada a nivel centro americano, es necesario descentralizarlo, y la solución para esto es incluir la virtualidad como puente entre los egresados que viven fuera del área metropolitana o que por horarios no tienen posibilidad de acceso a mantenerse al día en su profesión.

La universidad cuenta con los recursos y la disposición necesarios para desarrollar un programa virtual de educación continua.

Tenemos docentes dispuestos a "arriesgarse" capacitarse y dar el salto a esta nueva aventura ${ }^{3}$.

Estamos convencidos que sin duda un programa de educación continua virtual significaría un gran avance y aporte para nuestra profesión.

\section{Referencias Bibliográficas}

1. Berge ZL, Collins M, Dougherty K. Design Guidelines for Web-Based Courses. En: Abbey B, editor. Instructional and Cognitive Impacts of WebBased Education. Pensilvania, Estados Unidos: Idea Group Publishing; 2000. 32-41.

2. Bonk CJ, Cummings JA, Hara N, Fischler RB, Lee SM. A ten-level web integration continuum for higher education. En: Abbey B, editor. Instructional and Cognitive Impacts of Web-Based Education. Pensilvania, Estados Unidos: Idea Group Publishing; 2000. 56-78.

3. Lowther DL, Jones MG, Plants RT. Preparing tomorrow's teachers to use web-based education. En: Abbey B, editor. Instructional and Cognitive Impacts of Web-Based Education. Pensilvania, Estados Unidos: Idea Group Publishing; 2000. 129-147.
4. Maddux CD, Cummings R. Developing web pages as supplements to traditional courses. En: Abbey B, editor. Instructional and Cognitive Impacts of Web-Based Education. Pensilvania, Estados Unidos: Idea Group Publishing; 2000. 147-156.

5. Meyers PF. The HTML Web Classroom. Nueva Jerset, Estados Unidos: Prentice Hall; 1999.

6. Miller SM, Miller KL. Theoretical and practical considerations in the design of Web-based instruction. En: Abbey B, editor. Instructional and Cognitive Impacts of Web-Based Education. Pensilvania, Estados Unidos: Idea Group Publishing; 2000. 156-178.

7. Schutte JG. 1996. Virtual teaching in higher education: the new intellectual superhighway or just another traffic jam? Disponible en: https://pdfs. semanticscholar.org/6ad5/30196f652b9b480ad38d50eacd8b4cdbb7b3.pdf

8. Spiro RJ, Feltovich PJ, Jacobson MJ, Coulson RL. Knowledge representation, content specification, and the development of skill in situation specific knowledge assembly: Some constructivist issues as they relate to Cognitive Flexibility Theory and hypertext. En: Duffy TM, Jonassen DH, editores. Constructivism and the technology of instruction: a conversation. Nueva Jersey, Estados Unidos: Lawrence Erlbaum; 1992. 121-128. 